\title{
Tuning Neutron Resonance Spin-Echo Spectrometers with Pulsed Beams
}

\author{
Tatsuro Oda $\odot,{ }^{1, *}$ Masahiro Hino $\odot,{ }^{1}$ Hitoshi Endo, ${ }^{2}$ Hideki Seto $\odot,{ }^{2}$ and Yuji Kawabata ${ }^{1}$ \\ ${ }^{1}$ Institute for Integrated Radiation and Nuclear Science, Kyoto University, Kumatori, Osaka 590-0494, Japan \\ ${ }^{2}$ Institute of Materials Structure Science / J-PARC Center, High Energy Accelerator Research Organization, \\ Tokai, Ibaraki 319-1106, Japan
}

(Received 5 May 2020; revised 7 September 2020; accepted 22 October 2020; published 16 November 2020)

\begin{abstract}
The neutron spin-echo spectroscopy technique involving pulsed beams can be used to effectively access a wide range of space-time correlations of condensed matter. In this study, the features of this technique, in particular, the modulation of the intensity with zero effort (MIEZE) by using pulsed beams, which is based on the quantum-state manipulation of the neutron spin and energy, are comprehensively examined. A formulation of the MIEZE combined with the time of flight method (TOF MIEZE) is established by considering the characteristics of the pulsed neutron beams. Moreover, a parameter, namely, the detuning parameter, is introduced as a measure of the magnitude of detuning from the optimized instrumental state, known as the spin-echo condition. The phase and frequency shifts of the neutron intensity signals resulting from the TOF MIEZE under various configurations are investigated systematically. It is found that the detuning parameter equals the derivative of phase with respect to the TOF, whose zero-point corresponds to the spin-echo condition. The theoretical predictions on phase and frequency shifts by the established formulation are well validated by the experiments using an intense pulsed neutron source. The detuning parameter helps clarify the principle of the TOF MIEZE technique and can provide practical guidance regarding the implementation and optimization of spectrometers.
\end{abstract}

DOI: 10.1103/PhysRevApplied.14.054032

\section{INTRODUCTION}

Neutron spin echo (NSE) [1] represents a type of quasielastic neutron-scattering spectroscopy with a high resolution in energy. This approach can be used to directly observe the intermediate scattering function $\mathcal{I}(Q, t)$, which is the Fourier transform of the dynamic structure factor $\mathcal{S}(Q, \omega)$. Owing to its high resolution, the NSE spectroscopy approach has been successfully applied in the slow dynamics investigations in several domains ranging from polymer systems [2] to magnetism [3]. The modulation of intensity with zero effort (MIEZE) [4] is a type of neutron resonance spin-echo (NRSE) technique [5], in which resonance spin flippers (RSF) driven by a $\mathrm{rf}$ oscillating field are employed. Because all the neutronspin manipulations can be performed before the sample position, the MIEZE can be easily applied under various sample environments including high magnetic fields [6], which is hard to work with the conventional NSE method.

\footnotetext{
*t_oda@rri.kyoto-u.ac.jp

Published by the American Physical Society under the terms of the Creative Commons Attribution 4.0 International license. Further distribution of this work must maintain attribution to the author(s) and the published article's title, journal citation, and DOI.
}

Moreover, the MIEZE technique can be applied to perform the polarimetry analysis of scattered neutrons with additional spin analyzers [7].

These advantages of the MIEZE are especially useful to examine the spin dynamics. One of the promising scopes of MIEZE spectroscopy is the dynamical phenomena governed by very small energy exchange in nano to submicro structures, which are accessible through the small momentum transfer $Q$. Recently, the longitudinal-type resonance spin-echo spectrometer [8] at the research reactor neutron source at Heinz Maier-Leibnitz Zentrum (MLZ) used the MIEZE mode to analyze strongly correlated electron systems [9]. The critical dynamics near the Curie temperature in iron $[10,11]$ is an example. Several NSE and MIEZE measurements were performed on the phase transition from the paramagnetic to skyrmion lattice in $\mathrm{MnSi}$ under a magnetic field $[12,13]$. Recently, the MIEZE technique using pulsed neutron beam presented in this work was also applied to the same system [14]. The inherent fine wavelength resolution of the TOF approach permits the measurements of $\mathcal{I}(Q, t)$ in precisely divided regions of interest in the reciprocal space. This feature plays an important role in identifying whether the characteristic scattering pattern arising near phase boundary is derived from the static disorder or dynamical one, which pertains to the order stability and formation process. The analysis 
of $\mathcal{I}(Q, t)$ in finely resolved $Q$ regions provided a microscopic picture of the formation of the magnetic skyrmion lattice.

Accelerator-based spallation neutron sources such as ISIS, SNS, J-PARC, CSNS, and ESS have been established as alternatives to research reactors. Under such circumstances, NSE spectroscopy using the TOF method at the pulsed source has been studied [15-18] and applied [19] to cover a wide space-time range with the broadband wavelength. Several researchers have performed experiments using this combined approach on MIEZE, termed as TOF MIEZE, to establish the spectroscopic technique [20-23]. In general, to observe a MIEZE signal, the apparatus must be aligned to the spin-echo condition, in which the velocity-dependent phase shifts of neutrons are cancelled. However, the extremely narrow margin of instrumental alignment hinders the widespread implementation of MIEZE spectroscopy. In previous work, we examined the effect of the pulse width on the signal contrast by using pulsed beams simulated by a disk chopper at a steady-state neutron source [24]. It was noted that the decrease in contrast caused by the nondispersive deviation from the spin-echo condition was very small when using a short pulse width. In addition, the observed frequency shift was consistent with a parameter calculated considering the instrumental configuration. Although only one off-echo condition with a small deviation was considered in this experiment, the result indicating that the frequency shift, or the detuning parameter, can be a useful indicator to realize the tuning of a MIEZE instrument. In this regard, the objective of this study was to quantitatively verify the detuning parameter under various experimental conditions with much larger deviations. We further developed a formulation of TOF MIEZE technique considering its specific features that arise from the characteristics of pulsed neutron beams. The validity of the formulation was experimentally demonstrated with higher-quality TOF MIEZE signal data than reported previously using an intense spallation neutron source. It was found that, in addition to its role in rearranging the formulation, the detuning parameter corresponds to the derivative of phase with respect to TOF, which is a key quantity closely connected with the spin-echo condition. The effect of the deviation on the TOF MIEZE signals, particularly, in terms of the phase and frequency shifts, and the reduction in contrast were examined.

To maximize the advantage of the wide wavelength band available in a pulsed neutron source, it is necessary to cover a wide solid angle by using multiple detectors. However, unless the detectors have a perfect spherical surface centered on the sample, the signal phases may differ at different positions. Integrating the MIEZE signals with a certain momentum transfer $Q$ may cause a contrast reduction owing to the position-dependent phase shifts. The understanding of the phase shift of the TOF MIEZE signals leads to an accurate measurement of $I(Q, \tau)$ correcting the contrast reduction caused by instrument-related factors.

In this paper, the characteristics of the TOF MIEZE signals are discussed in detail, which are essential to perform NSE experiments with high precision.

\section{TOF MIEZE TECHNIQUE}

\section{A. Modulation of intensity with zero effort}

This section provides a brief review of the principle of MIEZE technique, based on a derivation reported in Ref. [24]. The MIEZE technique is used to generate a timebeating neutron intensity modulation, whose frequency is determined by the energy difference of the coherent neutron states produced by the RSF [25]. We consider a simple setup involving the MIEZE with two RSFs, as shown in Fig. 1. Both the RSFs are set to be in the $\pi / 2$-flip condition, in which the probability of spin flip is $1 / 2$. The angular frequencies of the first (RSF1) and second flipper (RSF2) are $\omega_{1}$ and $\omega_{2}$, respectively, with $\omega_{1}<\omega_{2}$. In the transition from the spin-up (down) to spin-down (up) state, the neutrons lose (gain) energy $\hbar \omega_{1}$. The interaction of the RSF and the principle of MIEZE has been described via plane-wave representation in Refs. [26-28]. Because the RSF manipulates the neutron state both in spin and energy [29], a notation involving the labels of the spin and energy is used to specify a state. For instance, the incident neutron is represented as $\left|\uparrow, \omega_{0}\right\rangle=\exp \left[i\left(k_{0} y-\omega_{0} t\right)\right]|\uparrow\rangle$. Here, $y$ and $t$ denote the spatial and time coordinate variable, respectively. Let $E_{0}$ denote the incident kinetic energy of the neutron in zero-field space, with $\omega_{0}=E_{0} / \hbar$. The wave number $k_{0}$ is defined as $k_{0}=\sqrt{2 m \omega_{0} / \hbar}=\sqrt{2 m E_{0} / \hbar^{2}}=$ $m v / \hbar$, where $v=\sqrt{2 E_{0} / m}$ is the neutron velocity, $m$ is the neutron mass, and $\hbar$ is the reduced Planck constant. $|\uparrow\rangle$ and $|\downarrow\rangle$ denote the spin-up and spin-down eigen states, respectively. Owing to the $\pi / 2$ flip in RSF1, a superposition state of the spin-up and spin-down components with different energies is created:

$$
\left|\uparrow, \omega_{0}\right\rangle \longrightarrow \frac{1}{\sqrt{2}}\left(\left|\uparrow, \omega_{0}\right\rangle+e^{i \chi_{1}}\left|\downarrow, \omega_{0}-\omega_{1}\right\rangle\right) .
$$

As the spin-up component experiences a spin flip from up to down at the resonant frequency $\omega_{1}$, its energy becomes $\hbar\left(\omega_{0}-\omega_{1}\right)$. The constant phase $\chi_{1}$ depends on the phase of the rf current of RSF1. In the travel from RSF1 to RSF2, the phase difference between $\left|\uparrow, \omega_{0}\right\rangle$ and $\left|\downarrow, \omega_{0}-\omega_{1}\right\rangle$ is accumulated, as follows [30]:

$$
\begin{gathered}
\phi_{12}=\int_{y_{1}}^{y_{2}}\left(k_{1}-k_{0}\right) d y \simeq-\frac{\omega_{1}}{v} L_{12}, \\
\text { with } k_{1}=\sqrt{\frac{2 m\left(\omega_{0}-\omega_{1}\right)}{\hbar}} \simeq k_{0}-\frac{\omega_{1}}{v} .
\end{gathered}
$$


Here, $k_{1}$ is the wave number of $\left|\downarrow, \omega_{0}-\omega_{1}\right\rangle$ state, and $L_{12}$ is the path length from RSF1 to RSF2. The approximation is derived from the fact that $\omega_{1} \ll \omega_{0}$. We assume that no magnetic field is present in the path $y_{1} \rightarrow y_{2}$. Then, the state of neutrons entering RSF 2 can be written as

$$
\frac{1}{\sqrt{2}}\left[\left|\uparrow, \omega_{0}\right\rangle+e^{i\left(\phi_{12}+\chi_{1}\right)}\left|\downarrow, \omega_{0}-\omega_{1}\right\rangle\right] .
$$

After the second $\pi / 2$ flip in RSF2, the following superposition state can be defined, consisting of four components [see Fig. 1(b)]:

$$
\begin{aligned}
& \frac{1}{2}\left[\left|\uparrow, \omega_{0}\right\rangle+e^{i\left(\phi_{12}+\chi_{1}+\chi_{2}\right)}\left|\uparrow, \omega_{0}-\omega_{1}+\omega_{2}\right\rangle\right. \\
& \left.\quad+e^{i\left(\phi_{12}+\chi_{1}\right)}\left|\downarrow, \omega_{0}-\omega_{1}\right\rangle+e^{i \chi_{2}}\left|\downarrow, \omega_{0}-\omega_{2}\right\rangle\right] .
\end{aligned}
$$

Here, $\chi_{2}$ is a constant that depends on the rf phase of RSF2. The first term, $\left|\uparrow, \omega_{0}\right\rangle$ is the nonflipped component. The second term, $\left|\uparrow, \omega_{0}-\omega_{1}+\omega_{2}\right\rangle$ corresponds to the resonant spin flips experienced both in RSF1 and RSF2. The third term $\left|\downarrow, \omega_{0}-\omega_{1}\right\rangle$ and fourth term $\left|\downarrow, \omega_{0}-\omega_{2}\right\rangle$ represent once-flipped components at RSF1 and RSF2, respectively. As the first two components in the spin-up state are spin analyzed and detected, only these components are considered in the following analyses.

On the path from RSF2 to the detector $y_{2} \rightarrow y_{d}$, the velocity-dependent phase difference, $\phi_{2 d}$ between $\left|\uparrow, \omega_{0}\right\rangle$ and $\left|\uparrow, \omega_{0}+\omega_{2}-\omega_{1}\right\rangle$ states must also be considered.

$$
\phi_{2 d}=\int_{y_{2}}^{y_{d}}\left(k_{M}-k_{0}\right) d y \simeq \frac{\omega_{2}-\omega_{1}}{v} L_{2 d},
$$

with $k_{M}=\sqrt{\frac{2 m\left(\omega_{0}-\omega_{1}+\omega_{2}\right)}{\hbar}} \simeq k_{0}+\frac{\omega_{2}-\omega_{1}}{v}$,

where $k_{M}$ is the wave number of the $\left|\uparrow, \omega_{0}-\omega_{1}+\omega_{2}\right\rangle$ state, and $L_{2 d}$ is the path length from RSF2 to the detector. Finally, the state of the neutron detected at $y=y_{d}, t=t_{d}$ can be expressed as follows, with respect to the relative phase:

$$
\frac{1}{2}\left\{1+e^{i\left[\phi_{12}+\phi_{2 d}+\chi-\left(\omega_{2}-\omega_{1}\right) t_{d}\right]}\right\} e^{i\left(k_{0} y_{d}-\omega_{0} t_{d}\right)}|\uparrow\rangle,
$$

where $t_{d}$ denotes the detection time, and $\chi=\chi_{1}+\chi_{2}$. The phase difference can be defined as a function of the neutron velocity $v$ and $t_{d}$ :

$$
\begin{aligned}
\phi\left(v, t_{d}\right) & =\phi_{12}(v)+\phi_{2 d}(v)-\left(\omega_{2}-\omega_{1}\right) t_{d}+\chi \\
& \simeq-\frac{\omega_{1}}{v} L_{12}+\frac{\omega_{2}-\omega_{1}}{v} L_{2 d}-\left(\omega_{2}-\omega_{1}\right) t_{d}+\chi .
\end{aligned}
$$

The detected neutron intensity is proportional to the following probability:

$$
\left|\frac{1}{2}\left(1+e^{i \phi\left(v, t_{d}\right)}\right)\right|^{2}=\frac{1+\cos \phi\left(v, t_{d}\right)}{2} .
$$

(a)

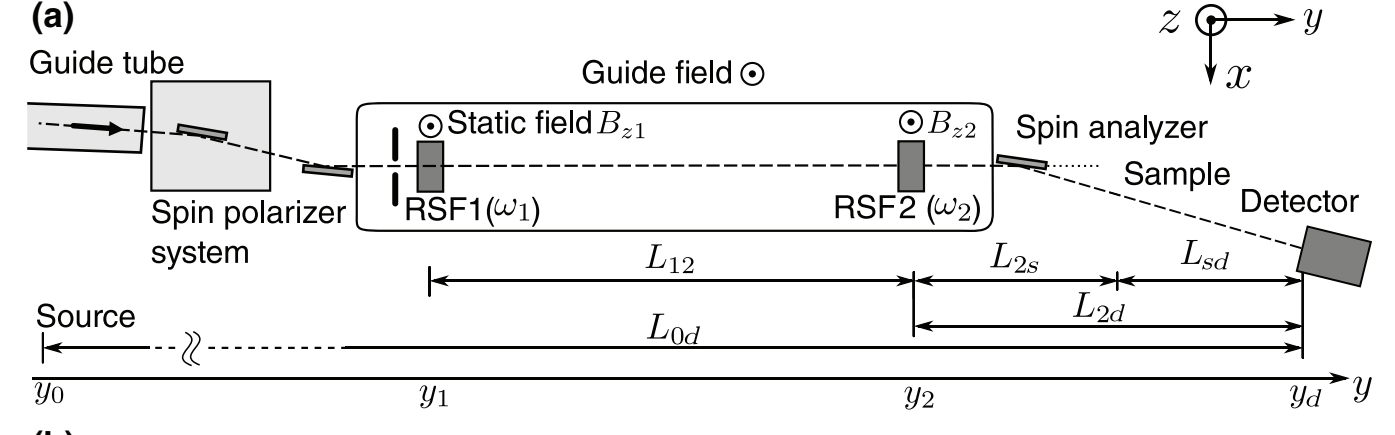

(b)

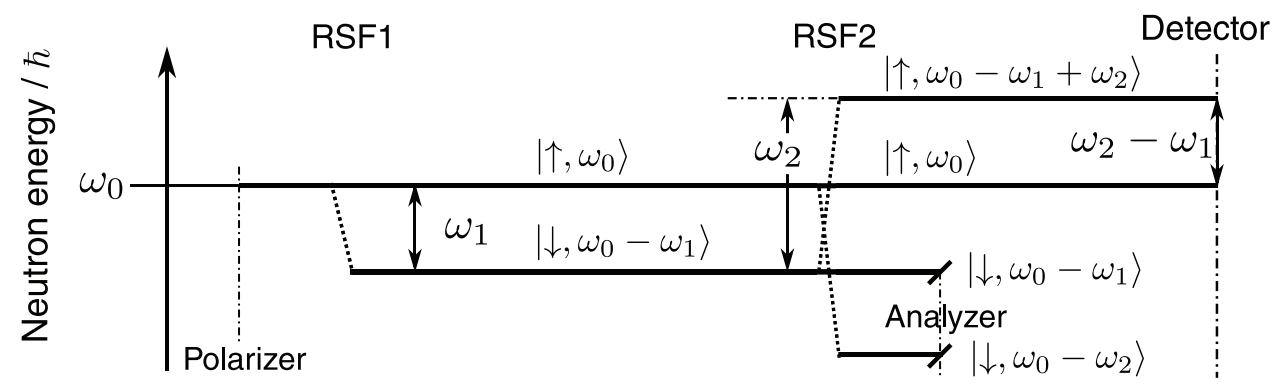

FIG. 1. (a) Schematic geometry of the MIEZE instrument at BL06 at MLF, J-PARC. (b) Neutron energy diagram for spin manipulation with two $\pi / 2$ RSFs. 
As $\phi\left(v, t_{d}\right)$ depends on the neutron velocity, in general, Eq. (10) does not exhibit any clear sinusoidal oscillations. If the velocity dependence of $\phi\left(v, t_{d}\right)$ can be eliminated by tuning the apparatus, the phase becomes $\phi\left(t_{d}\right)=$ $-\left(\omega_{2}-\omega_{1}\right) t_{d}+\chi$, and the intensity oscillates proportionally to $1+\cos \phi\left(t_{d}\right)=1+\cos \left[\left(\omega_{2}-\omega_{1}\right) t_{d}-\chi\right]$, independent from the incident beam monochromaticity. The frequency of the time oscillation of MIEZE corresponds to the energy difference determined using RSF1 and RSF2. The MIEZE frequency is expressed as $\omega_{M}=\omega_{2}-\omega_{1}$. The cancellation of the velocity dependence allows for a wide spectrum acceptance for MIEZE signals, similar to in the conventional NSE method. The spin-echo condition for the MIEZE instrument, which is termed as the MIEZE condition, can be expressed as

$$
\omega_{1} L_{12}=\omega_{M} L_{2 d}
$$

Thus, in the MIEZE technique, the cancelation of the velocity-dependent phase is realized by tuning the apparatus, specifically, the distances between the spin flippers and the detector, and the resonant frequencies. In this condition, we measure a time-modulation of the neutron intensity with the frequency $\omega_{M}$,

$$
I\left(t_{d}\right) \propto \frac{1+\cos \left(\omega_{M} t_{d}-\chi\right)}{2} .
$$

Note that the MIEZE system can include several combinations of $\pi$ and $\pi / 2$ flippers [31]. The $\pi / 2-\pi-\pi-\pi / 2$ configuration with four spin flippers yields a double MIEZE frequency, that is, $\omega_{M}=2\left(\omega_{2}-\omega_{1}\right)$. In the following analyses, $\chi$ is omitted as it gives only a constant phase shift of the MIEZE signals.

\section{B. MIEZE spectroscopy for quasielastic neutron scattering}

When the neutron velocity changes from $v \rightarrow v+\delta v$ through quasielastic scattering by a sample, in the echo condition, the phase is modified to [24]

$$
\phi\left(t_{d}, v+\delta v\right) \simeq-\omega_{M} t_{d}+\frac{\omega_{M} L_{s d}}{v^{2}} \delta v
$$

This represents the first-order approximation in $\delta v / v$. In most spin-echo measurements, $\delta v / v$ is typically of the order from $10^{-5}$ to $10^{-3}$. Let $\hbar \omega$ denote a neutron energy change corresponding to a velocity change of $\delta v$. Omitting the higher-order terms of $\delta v$, the following expression can be obtained: $\hbar \omega=(m / 2)\left[(v+\delta v)^{2}-v^{2}\right] \simeq m v \delta v$. Using this relation, $\phi\left(t_{d}, v+\delta v\right)$ can be rewritten in terms of the energy exchange $\omega$ :

$$
\phi\left(t_{d}, v+\delta v\right) \simeq-\omega_{M} t_{d}+\omega \tau,
$$

with

$$
\tau=\frac{\hbar \omega_{M} L_{s d}}{m v^{3}}
$$

Here, $\tau$ has dimensions of time and is known as the Fourier time or spin-echo time. The Fourier time represents the correlation time of the motions of interest in NSE spectroscopy.

The probability of occurrence of a scattering that involves a momentum transfer $Q$ and an energy transfer $\hbar \omega$ is given by the dynamic structure factor $\mathcal{S}(Q, \omega)$ [32]. Considering the occurrence of such a scattering event, the intensity of the scattered neutrons at a given $Q$ and $\omega$ is [33]

$$
\begin{aligned}
I\left(t_{d}\right) & \propto \frac{1}{2} \int d(\hbar \omega) \mathcal{S}(Q, \omega)\left[1+\cos \left(-\omega_{M} t_{d}+\omega \tau\right)\right] \\
& \propto \frac{1}{2}\left[1+\mathcal{I}(Q, \tau) \cos \left(\omega_{M} t_{d}\right)\right]
\end{aligned}
$$

where $\mathcal{I}(Q, \tau)=\int d(\hbar \omega) \mathcal{S}(Q, \omega) \cos (\tau \omega)$ is equivalent to the real part of the intermediate scattering function. We assume that $\mathcal{S}(Q, \omega)$ is an even function with respect to $\omega$, which is valid for the quasielastic neutron scattering. Equation (16) indicates that the contrast of the intensity modulation decreases by a factor of $\mathcal{I}(Q, \tau)$ owing to the change in energy of the neutron. By observing the contrast reduction of MIEZE signals, the dynamical information of a sample can be obtained in the form of $\mathcal{I}(Q, \tau)$. By using pulsed beams with a broad spectrum of neutron wavelength $\lambda$, a wide area of $(Q, \tau)$ space is covered as they vary in accordance with $Q \propto \lambda^{-1}$ and $\tau \propto \lambda^{3}$.

\section{Features of TOF MIEZE}

This section discusses the specific features of the TOF MIEZE technique in the context of a pulsed neutron source. The neutron velocity $v$ can be determined using the TOF approach, as follows:

$$
v=\frac{L_{0 d}}{t_{0 d}},
$$

where $L_{0 d}$ is the length of the total flight path from the source to the detector, and $t_{0 d}$ is the TOF defined as $t_{0 d}=$ $t_{d}-t_{0}$ with a detection time of $t_{d}$ and pulse generation time of $t_{0}$. In the spin-echo condition, the resultant phase differences of the neutrons are independent of their velocities, i.e., $\partial \phi / \partial v=0$ [34]. The TOF, $t_{0 d}$, is the very measurand primarily recorded in pulsed-beam experiments. Thus, we 
consider the derivative of the phase with respect to the TOF instead of the velocity, as follows:

$$
\frac{\partial \phi\left(v, t_{d}\right)}{\partial t_{0 d}}=\frac{\partial \phi\left(v, t_{d}\right)}{\partial v} \frac{\partial v}{\partial t_{0 d}}=\frac{-\omega_{1} L_{12}+\omega_{M} L_{2 d}}{L_{0 d}} .
$$

The right-hand side equals a parameter that we introduced as detuning frequency in our previous report [24]. Specifically, the detuning parameter indicates the magnitude of the deviation from the echo condition, as specified in Eq. (11). In the remaining paper, the parameter is denoted as $\Omega$ :

$$
\Omega=\frac{-\omega_{1} L_{12}+\omega_{M} L_{2 d}}{L_{0 d}} .
$$

Substituting the expression into Eq. (9) yields

$$
\phi\left(v, t_{d}\right)=-\omega_{M} t_{d}+\Omega \frac{L_{0 d}}{v},
$$

or

$$
\phi\left(t_{0}, t_{d}\right)=-\omega_{M} t_{d}+\Omega t_{0 d}=-\left(\omega_{M}-\Omega\right) t_{d}+\Omega t_{0} .
$$

When the pulse generation time $t_{0}$ is fixed, $\Omega t_{0}$ corresponds to a constant phase. Equation (21) indicates that the velocity-dependent phase shift $\Omega L_{0 d} / v$ results in a frequency shift of a TOF MIEZE signal by $\Omega$. It should be noted that $\Omega$ can be interpreted as the derivative of the phase with respect to the TOF, $\partial \phi\left(v, t_{d}\right) / \partial t_{0 d}$, as shown in Eq. (18). The equality between the frequency shift and the phase derivative is examined, as described in the experimental section.

The dephasing effect owing to the uncertainty of the detection time, $\Delta t_{d}$ limits the resolution of the MIEZE instrument. For a MIEZE signal with a frequency of the order of megahertz, a high precision of $t_{d}$, which is considerably less than $1 \mu \mathrm{s}$ is required in addition to the satisfaction of the echo condition. This requirement pertaining to the time-resolution restricts the thickness of the detector. More importantly, this requirement limits the sample volume and shape [35-38]. Although $\Delta t_{d}$ is likely a significant factor in most scattering experiments, in the present study we focus on the dephasing effect associated with $\Delta t_{0}$, which is known as the pulse width and is a fundamental parameter characterizing a neutron pulse beam, to investigate the characteristics of TOF MIEZE signals. In pulsed sources, the wavelength resolution $\Delta \lambda / \lambda$ is equivalent to $\Delta t_{0 d} / t_{0 d}$, and it is considerably smaller than the ordinary resolution of quasimonochromatic beams $(\Delta \lambda / \lambda \sim 10 \%)$ used in NSE instruments at steady-state sources. The inherent fine wavelength resolution of a pulsed beam allows the retention of the signal contrast even when the system is deviated from the echo condition.

\section{EXPERIMENT DETAILS}

The experiment is performed using a MIEZE instrument constructed at neutron beam line No. 6 (BL06) at the Materials and Life Science Experimental Facility (MLF) [39-41] of the Japan Proton Accelerator Research Complex (J-PARC). The spallation source of the MLF produces intense pulsed neutron beams with a broad spectrum optimized with respect to cold neutrons via the liquid hydrogen moderator [42]. The BL06 has two beam ports for neutron resonance spin-echo spectrometer and MIEZE instruments [43].

In the experiment, the simplest MIEZE system, which consisted of two $\pi / 2$ flippers, is used. The schematic geometry of the MIEZE system is shown in Fig. 1. The frequencies of the first (RSF1) and second (RSF2) flipper are set as $\omega_{1}$ and $\omega_{2}$, respectively, and the MIEZE frequency is thus $\omega_{M}=\omega_{2}-\omega_{1}$. To inspect the properties of the TOF MIEZE signals, several configurations are considered for the MIEZE instrument at BL06, as summarized in Table I. Instead of the angular frequency, we use $f_{i}=\omega_{i} / 2 \pi(i=$ $1,2, M)$ to express the values of the frequency. To extend the available neutron wavelength band, the second frame mode, in which the pulse repetition frequency is $12.5 \mathrm{~Hz}$ is employed, with the original mode corresponding to $25 \mathrm{~Hz}$. At the outlet of the neutron guide tube, the neutrons are polarized by a supermirror made of $\mathrm{Fe} / \mathrm{SiGe}$ multilayers. The analyzer is a supermirror with the same characteristics as the polarizer. The time- and position-sensitive detector is composed of a scintillator and a photomultiplier tube [44]. Because of the prerequisite of time resolution to measure a high-frequency MIEZE signal, a thin ${ }^{6} \mathrm{Li}$-glass scintillator with a thickness of $0.2 \mathrm{~mm}$ is used. By comparison with a ${ }^{3} \mathrm{He}$ detector, the neutron detection efficiency is evaluated to be over $50 \%$ for the neutron wavelengths longer than $0.35 \mathrm{~nm}$. The spatial resolution of the detector is less than $1.0 \mathrm{~mm}$. The data acquisition system is based on the NEUNET module developed by the High Energy Accelerator Research Organization (KEK) [45]. The neutron detection event is recorded in the event-data format, in which the individual detection event included the information of the TOF, detected position, pulse height, and absolute time stamp.

The stationary field $B_{z}$ of the RSF is applied in the $z$ direction, as shown in Fig. 1(a). An oscillating field orthogonal to $B_{z}$ is generated along the $x$ direction. Under the

TABLE I. Instrumental parameters of MIEZE configurations (i), (ii), and (iii).

\begin{tabular}{lcccccc}
\hline \hline & $f_{M}$ & $f_{1}$ & $f_{2}$ & & & \\
& $(\mathrm{kHz})$ & $(\mathrm{kHz})$ & $(\mathrm{kHz})$ & $L_{12}(\mathrm{~m})$ & $L_{2 d}(\mathrm{~m})$ & $L_{0 d}(\mathrm{~m})$ \\
\hline (i) & 200 & 200 & 400 & 2.88 & 2.39 & 23.60 \\
(ii) & 200 & 200 & 400 & 1.15 & 0.66 & 20.54 \\
(iii) & 20 & 50 & 70 & 0.86 & 1.23 & 20.70 \\
\hline \hline
\end{tabular}


magnetic resonance condition, the spin-flip probability is $P=\sin ^{2}\left[|\mu| B_{r} \ell /(\hbar v)\right]$, where $v$ is the neutron velocity, $B_{r}$ is the amplitude of the oscillating magnetic field, $\ell$ is the length of the rf coil, and $\mu$ is the neutron magnetic moment. The cases with $P=1$ and $P=1 / 2$ are termed as $\pi$ flip and $\pi / 2$ flip, respectively. To ensure a constant flip probability for a wide range of the neutron spectra, the amplitude $B_{r}$ is changed inversely with the TOF [46].

$$
B_{r}\left(t_{0 d}\right)=\frac{\pi}{2} \beta \frac{\hbar L}{|\mu| \ell} \frac{1}{t_{0 d}},
$$

where $L$ is the distance from the source to the flippers. In addition, $\beta=1$ for $\pi$ flip, and $\beta=1 / 2$ for $\pi / 2$ flip. Figure 2(a) shows the data used to tune the $\pi$ flip of a RSF with a frequency of $200 \mathrm{kHz}$ at fixed amplitudes $B_{r}$ presented as input $\mathrm{rf}$ voltages. The flipping ratio indicates the ratio of the neutron intensity when a $\pi$ flipper is on to that when it is off, that is, $I_{\mathrm{on}} / I_{\mathrm{off}}$. By using a time-varying $B_{r}$, a spin-flip efficiency of more than $90 \%$ (flipping ratio $>19$ ) is attained for a wide wavelength band as shown in Fig. 2(b).
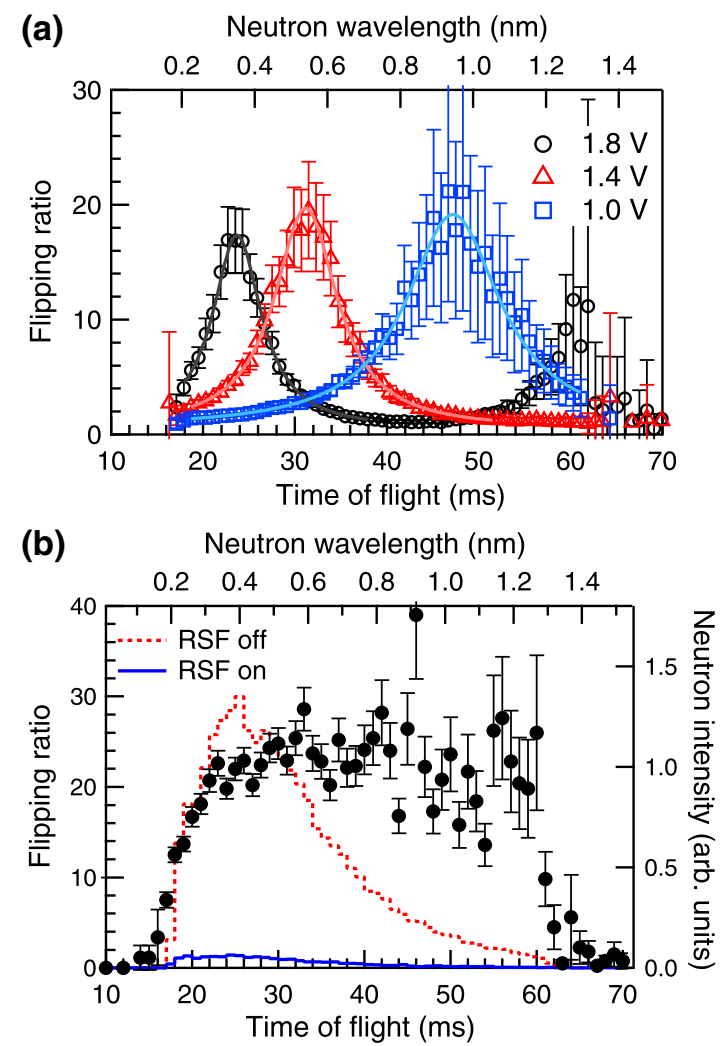

FIG. 2. (a) TOF dependence of the spin-flipping ratios at fixed input $\mathrm{rf}$ amplitudes, which are proportional to $B_{r}$. The solid lines indicate the best-fit curves by the Lorentzian function. (b) Spinflipping ratio for a pulsed white beam (black circles). The red dashed and blue solid lines indicate the TOF spectrum when the $\pi$ flipper is on and off, respectively.

\section{RESULTS AND DISCUSSION}

\section{A. TOF MIEZE signal}

Figures 3 and 4 show the raw TOF spectra containing MIEZE oscillations with frequencies of $f_{M}=200$ and $20 \mathrm{kHz}$ in the spin-echo conditions, respectively. The Fourier transforms of the TOF spectra show clear peaks at the expected frequencies, 200 and $20 \mathrm{kHz}$ as shown in Figs. 3(b) and 4(b), respectively. The time window of the Fourier transform is the entire time frame of TOF, 80 $\mathrm{ms}$, and thus, the frequency resolution is $12.5 \mathrm{~Hz}$. Panels (c)-(e) show the magnified individual MIEZE oscillations, at representative TOF (wavelength) ranges of $20 \mathrm{~ms}(\lambda \approx$ $0.4 \mathrm{~nm}), 40 \mathrm{~ms}(\lambda \approx 0.8 \mathrm{~nm})$, and $60 \mathrm{~ms}(\lambda \approx 1.2 \mathrm{~nm})$.
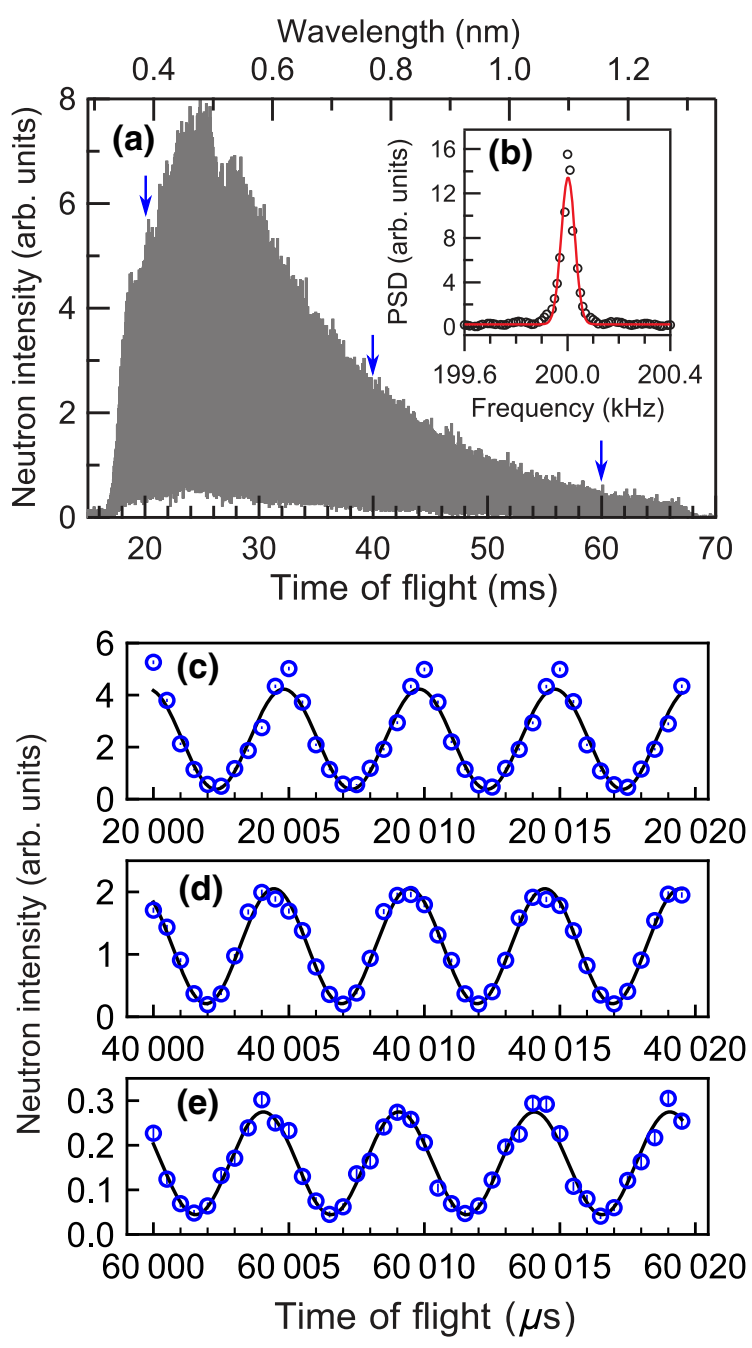

FIG. 3. (a) A 200-kHz TOF MIEZE signal in the echo condition. The time bin is $1 \mu \mathrm{s}$. The blue arrows indicate the points of TOF (wavelength) shown in (c)-(e). (b) Power spectral density (PSD) obtained by the Fourier transform of the TOF MIEZE signal. Panels (c)-(e) show the individual MIEZE oscillations at (c) TOF $20 \mathrm{~ms}(\lambda=0.385 \mathrm{~nm})$, (d) TOF $40 \mathrm{~ms}(\lambda=0.770 \mathrm{~nm})$, and (e) TOF $60 \mathrm{~ms}(\lambda=1.156 \mathrm{~nm})$ for $L_{0 d}=20.54 \mathrm{~m}$. The time bin is $0.5 \mu \mathrm{s}$. 

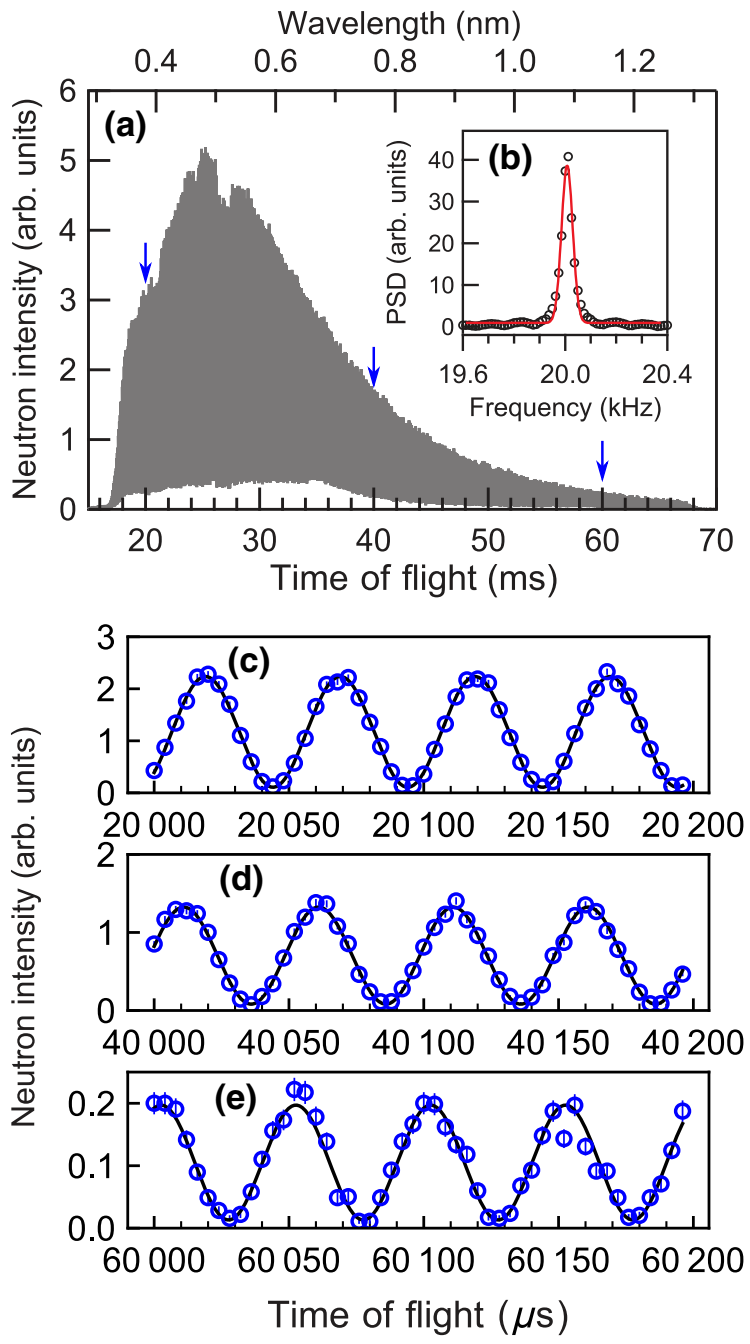

FIG. 4. (a) A 20-kHz TOF MIEZE signal in the echo condition. The time bin is $1 \mu \mathrm{s}$. The blue arrows indicate the magnified points of TOF (wavelength) shown in (c)-(e). (b) PSD obtained by the Fourier transform of the TOF MIEZE signal. Panels (c)-(e) show the individual MIEZE oscillations at (c) TOF 20 $\mathrm{ms}(\lambda=0.382 \mathrm{~nm}),(\mathrm{d})$ TOF $40 \mathrm{~ms}(\lambda=0.764 \mathrm{~nm})$, and (e) TOF $60 \mathrm{~ms}(\lambda=1.147 \mathrm{~nm})$ for $L_{0 d}=20.70 \mathrm{~m}$. The time bin is $5 \mu \mathrm{s}$.

\section{B. Phase and frequency shift induced by magnetic fields}

The presence of a magnetic field applied in the path from RSF1 to RSF2 may cause a deviation from the echo condition. In general, the phase difference owing to the momentum split, $\phi_{12}$, between the $\left|\uparrow, \omega_{0}\right\rangle$ and $\left|\downarrow, \omega_{0}-\omega_{1}\right\rangle$ states is modified by the magnetic potential $\mp|\mu| B$ (the spin-up and spin-down state takes - and + sign, respectively) in the flight path along the $y$ axis, as follows:

$$
\phi_{12}=\int_{y_{1}}^{y_{2}}\left(k_{1}^{-}-k_{0}^{+}\right) d y \simeq-\frac{\omega_{1}-\bar{\omega}}{v} L_{12}
$$

where

$$
\begin{gathered}
k_{0}^{+}=\sqrt{\frac{\omega_{0}-|\mu| B}{\hbar}} \simeq k_{0}-\frac{|\mu| B}{\hbar v}, \\
k_{1}^{-}=\sqrt{\frac{\omega_{0}-\omega_{1}+|\mu| B}{\hbar}} \simeq k_{0}-\frac{\hbar \omega_{1}-|\mu| B}{\hbar v},
\end{gathered}
$$

and

$$
\bar{\omega}=\frac{2|\mu|}{\hbar} \frac{1}{L_{12}} \int_{y_{1}}^{y_{2}} B(y) d y .
$$

The angular frequency $\bar{\omega}$ corresponds to the magnitude of the averaged magnetic field between RSF1 and RSF2. When the magnetic field is applied, the accumulated phase difference of the zero-field case $\phi_{12}=-\omega_{1} L_{12} / v$ [Eq. (2)] reduces to $\phi_{12}=-\left(\omega_{1}-\bar{\omega}\right) L_{12} / v$ [Eq. (23)]. Thus, in the presence of a magnetic field $\bar{\omega}$, the detuning parameter $\Omega$ is corrected to

$$
\Omega=\frac{-\left(\omega_{1}-\bar{\omega}\right) L_{12}+\omega_{M} L_{2 d}}{L_{0 d}} .
$$

The degree of freedom corresponding to the reduced $\phi_{12}$ allows for a certain flexibility in configuring the echo condition, especially for low $\omega_{M}$, which leads to a smaller Fourier time. In this regard, the concept of effective field integral subtraction, which was proposed by Häußler et al. $[47,48]$, has been exploited in longitudinal-type resonance spin-echo instruments to extend the dynamic range toward fast dynamics [9].

In the experimental setup at BL06, a weak magnetic guide field of approximately $0.45 \mathrm{mT}$ is applied in the region from the spin polarizer to the analyzer. A dipole magnet is used to generate the stationary field of the RSF. In this case, a certain amount of the field leaked outside the RSF on the neutron-beam path. The disagreement between the setup parameters listed in Table I and those of the MIEZE condition in the zero-field case [Eq. (11)] is due to the presence of nonzero magnetic fields.

To realize a deviation from the spin-echo condition, an additional coil is installed between RSF1 and RSF2, as depicted in Fig. 5(a). The angular frequency $\bar{\omega}$ is approximately estimated as $\bar{\omega}=(2|\mu| / \hbar)\left(L_{\text {coil }} / L_{12}\right) B_{\text {coil }}$ with the length and field of the coil being $L_{\text {coil }}$ and $B_{\text {coil }}$, respectively. The actually observed frequency of the TOF MIEZE signals is termed as the effective frequency, $f_{\text {eff }}=\omega_{\text {eff }} / 2 \pi$, which is expected to be $f_{\text {eff }}=f_{M}-\Omega / 2 \pi$, as expressed by Eq. (21). Figure 5(b) shows the frequency shifts induced by the magnetic fields of the additional coil. The magnitude of magnetic field is parameterized by $\bar{\omega}$. The value of $f_{\text {eff }}$ was experimentally determined by fitting a peak in the power spectral density with a Gaussian function. The 


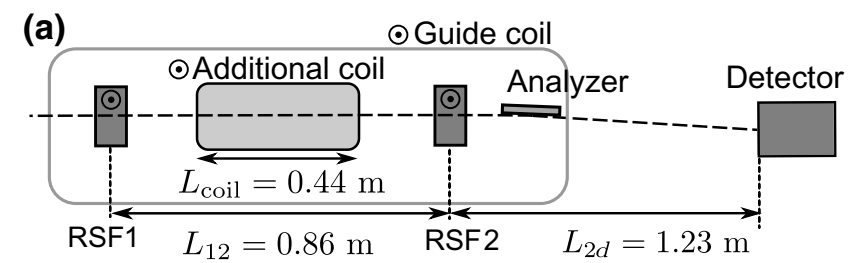

(b) Current of the additional coil (A)

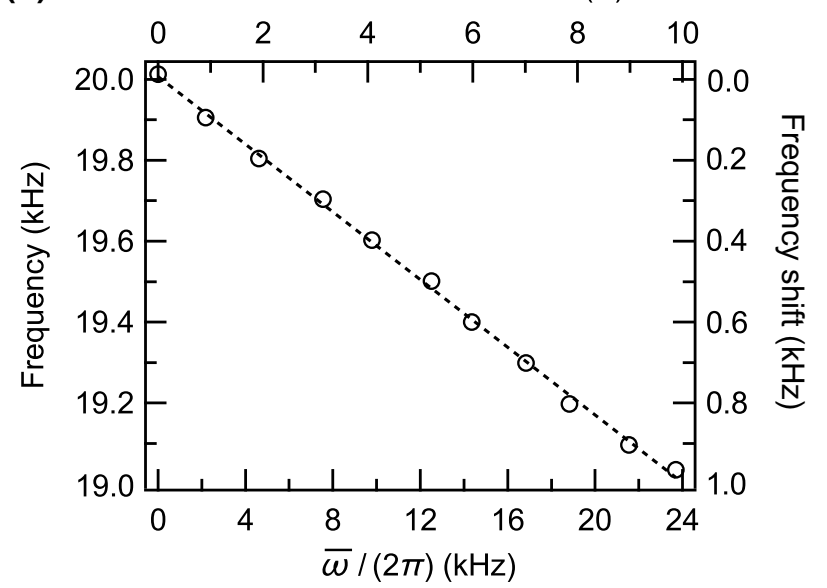

FIG. 5. (a) Setup with an additional magnet coil. Not in scale. (b) Frequency shifts (solid circle) from $f_{M}=20 \mathrm{kHz}$ in off-echo conditions induced by magnetic fields. The data corresponded to configuration (iii). $\bar{\omega}$ indicates the angular frequency corresponding to the averaged magnetic field in the path from RSF1 to RSF2, as expressed in Eq. (26). The dashed line indicates the best-fit curve.

frequency shift is defined as $f_{M}-f_{\text {eff }}$ and fitted using a linear function $f(x)=a x+b$, where $f$ denotes the frequency shift, and $x$ denotes $\bar{\omega} / 2 \pi$. The best-fit parameters are $a=$ $0.0419 \pm 0.001$ and $b=-0.007 \pm 0.007 \mathrm{kHz}$. The derivative of $\Omega$ with respect to $\bar{\omega}$ is calculated using Eq. (27), as

$$
\frac{\Delta \Omega}{\Delta \bar{\omega}}=\frac{L_{12}}{L_{0 d}}
$$

The experimental result for the slope $a=0.0419 \pm 0.001$ is in good agreement with the value $L_{12} / L_{0 d}=0.0415 \mathrm{cal}-$ culated using the setup parameters for configuration (iii).

\section{Phase and frequency shift induced by spatial displacements}

Equation (18) indicates that the derivative of the phase with respect to TOF equals the parameter $\Omega$, which can be detected as a frequency shift. To verify this aspect, we compare the observed frequency shift and the phase derivative of the TOF MIEZE signals. The phase derivative is obtained as a slope of the observed TOF dependency curve of the phase. In this case, deviations from the echo condition are realized by changing the position of the detector.
TABLE II. Frequency shifts and derivative of the phase with respect to the time of flight under off-echo conditions.

\begin{tabular}{lccc}
\hline \hline & $\begin{array}{c}\text { Displacement } \\
\Delta y_{d}(\mathrm{~mm})\end{array}$ & $\begin{array}{c}\text { Frequency shift } \\
f_{M}-f_{\text {eff }}(\mathrm{Hz})\end{array}$ & $\begin{array}{c}\text { Slope } \\
\Delta \phi / \Delta t_{0 d} / 2 \pi(\mathrm{Hz})\end{array}$ \\
\hline (a) & -10.0 & $80.2 \pm 0.2$ & $80.25 \pm 0.13$ \\
(b) & -4.0 & $31.7 \pm 0.2$ & $31.75 \pm 0.05$ \\
(c) & -0.6 & $4.5 \pm 0.2$ & $4.49 \pm 0.03$ \\
(d) & 0.0 & $-0.2 \pm 0.2$ & $-0.28 \pm 0.01$ \\
(e) & 0.6 & $-4.9 \pm 0.2$ & $-4.97 \pm 0.03$ \\
(f) & 4.0 & $-32.4 \pm 0.2$ & $-32.60 \pm 0.07$ \\
(g) & 10.0 & $-82.4 \pm 0.3$ & $-82.41 \pm 0.20$ \\
\hline \hline
\end{tabular}

Figure 6 shows the observed frequency shifts and TOF dependency of the phase of TOF MIEZE signals, under various $\Delta y_{d}$, which corresponds to the displacements from the echo condition, as described in Table II. The phase of the TOF MIEZE signals are shown in the range from $-\pi$ to $\pi$. The data are obtained using configuration (i). In the echo condition, (d) $\Delta y_{d}=0 \mathrm{~mm}$, the phase is nearly constant over the entire TOF frame. In the deviant conditions, the phases of the TOF MIEZE signal change linearly with the TOF. The coefficient of proportionality (slope of phase $\left.\Delta \phi / \Delta t_{0 d}\right)$ increases as the spatial displacement increases. Figure 6(h) presents a comparison of the frequency shifts and the slopes for all the deviant conditions (a)-(g). The frequency shifts are obtained by using the Fourier transform of the TOF MIEZE signal. The slopes are obtained via line fitting to the phase versus the TOF plot. The findings, including the sign of the slope, exhibit perfect agreement within the frequency resolution of the Fourier transform. The equivalence between the derivative of the phase with respect to the TOF and the frequency shift of the TOF MIEZE signals can thus be established.

Moreover, the equivalence between $\Omega$ and the frequency shift is examined in a wider range of frequency shift of the order of kilohertz. The echo condition described in Eq. (11) depends on the RSF frequencies, $\omega_{1}, \omega_{2}$ and distances $L_{12}$, $L_{2 d}$. To investigate the effect of the displacements on the TOF MIEZE signals, the distances are adjusted by changing the positions of RSF1, RSF2, and the detector, i.e., $y_{1}, y_{2}$, and $y_{d}$, respectively. The spatial coordinates are illustrated in Fig. 7 (a). $\Omega$ in the case with the displacement can be derived using Eq. (19). The line slope is the experimental result, which is compared with the value of $\Delta \Omega / \Delta y$ calculated analytically. In the case involving a displacement of RSF1, that is, $\Delta y_{1}$ from the echo condition, the position changes from $y_{1} \rightarrow y_{1}+\Delta y_{1}$, and the distance changes from $L_{12} \rightarrow L_{12}-\Delta y_{1}$. From Eq. (27), the derivative of $\Omega$ with respect to $y_{1}$ is

$$
\frac{\Delta \Omega}{\Delta y_{1}}=\frac{\omega_{1}-\bar{\omega}}{L_{0 d}} .
$$



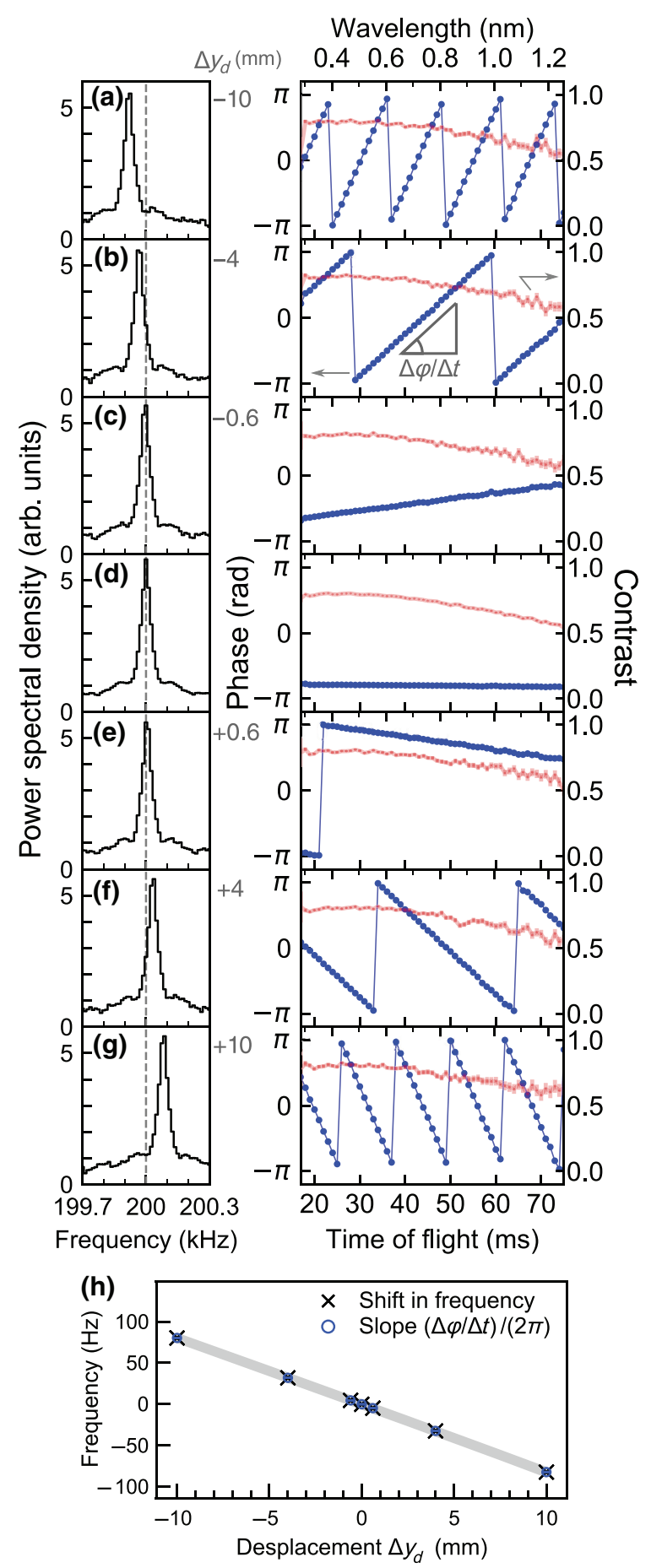

FIG. 6. Observed frequency, phase, and contrast of TOF MIEZE signals in various off-echo conditions with $\Delta y_{d}=$ $-10 \mathrm{~mm}$ (a), $-4.0 \mathrm{~mm}$ (b), $-0.6 \mathrm{~mm}$ (c), $0.0 \mathrm{~mm}$ (d), 0.6 $\mathrm{mm}$ (e), $4.0 \mathrm{~mm}$ (f), $10 \mathrm{~mm}$ (g). The left column shows the Fourier transforms of TOF MIEZE signal. The right column shows the TOF dependency of the phase (blue closed circles) and contrast (red lines). (h) Frequency shifts (cross marks) and slope, $\Delta \phi / \Delta t_{0 d} / 2 \pi$ (blue circles) in conditions (a)-(g). The gray shaded area corresponds to a frequency resolution of $12.5 \mathrm{~Hz}$, as determined by the time window of $80 \mathrm{~ms}$ used in the Fourier transform. The data are obtained using configuration (i).
Similarly, the derivatives of $\Omega$ with respect to $y_{2}$ and $y_{d}$ can be obtained:

$$
\begin{gathered}
\frac{\Delta \Omega}{\Delta y_{2}}=-\frac{\omega_{2}-\bar{\omega}}{L_{0 d}}, \\
\frac{\Delta \Omega}{\Delta y_{d}}=\frac{\omega_{M}}{L_{0 d}},
\end{gathered}
$$

where $\bar{\omega}$ is the angular frequency corresponding to the average magnetic field between RSF1 and RSF2, as defined by Eq. (26). In the setup, a guide field of approximately $0.45 \mathrm{mT}$ was applied in $L_{12}$. Subsequently, $\bar{\omega} / 2 \pi$ is estimated to be $13 \mathrm{kHz}$. Figure 7(b) shows the frequency shifts for various spatial displacements. The frequency shift is defined as $f_{M}-f_{\text {eff. }}$. The slope is obtained by fitting with a line $f(x)=a x+b$, where $f$ denotes the frequency shift, and $x$ denotes the displacements $\left(\Delta y_{1}, \Delta y_{2}\right.$, or $\left.\Delta y_{d}\right)$. The obtained slopes $a$ are compared with the calculated $\Delta \Omega / \Delta y_{i}$ presented in Table III. The two values are noted to be in agreement. For the fitting model, the intercept $b$ is

(a)

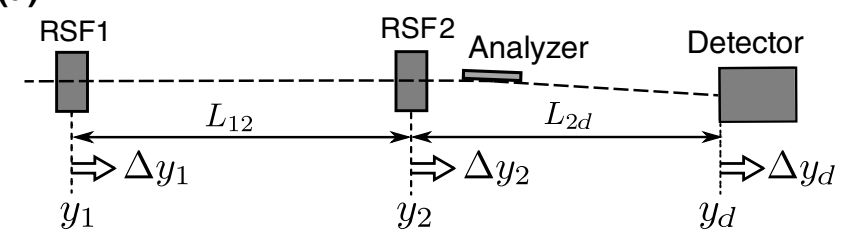

(b) Displacement of RSF2 $\Delta y_{2}(\mathrm{~mm})$

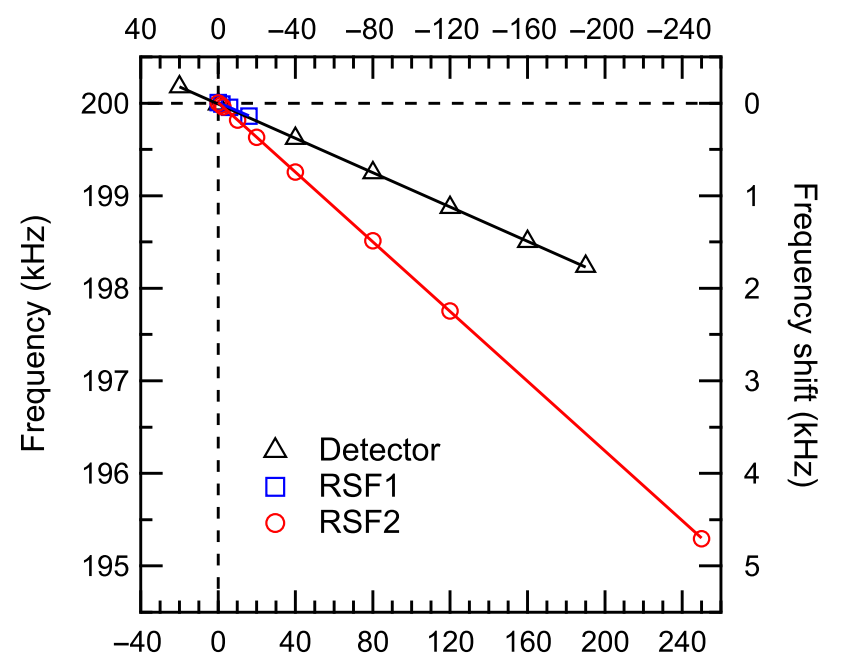

Displacement of RSF1 $\Delta y_{1}$ or Detector $\Delta y_{d}(\mathrm{~mm})$

FIG. 7. (a) Displacements of the components. (b) Frequency shift induced by the spatial displacement of RSF1 (square), RSF2 (circle), and the detector (triangle). The displacement of RSF2 is displayed inversely to the others. The data corresponded to configuration (ii). The solid lines exhibit the best-fit curves for each data set. 
TABLE III. Experimental results of the line slope $a$ of the frequency shift versus the displacement plot and $\Delta \Omega / \Delta y_{i}$ calculated using Eqs. (29), (30), and (31).

\begin{tabular}{lcc}
\hline \hline & Experiment & Calculation \\
Component & Slope $a(\mathrm{~Hz} / \mathrm{mm})$ & $\Delta \Omega / \Delta y_{i} / 2 \pi(\mathrm{Hz} / \mathrm{mm})$ \\
\hline RSF1 & $9.05 \pm 0.09$ & 9.10 \\
RSF2 & $-18.83 \pm 0.03$ & -18.84 \\
Detector & $9.27 \pm 0.02$ & 9.74 \\
\hline \hline
\end{tabular}

expected to be zero. The fitting results are $b=-0.007 \pm$ $0.001 \mathrm{kHz}$ for RSF1, $b=0.008 \pm 0.003 \mathrm{kHz}$ for RSF2, and $b=0.010 \pm 0.002 \mathrm{kHz}$ for the detector. The results are reasonably close to zero considering the frequency resolution of the Fourier transform, $0.0125 \mathrm{kHz}$.

\section{Contrast reduction}

Figure 8 shows the experimental results pertaining to the contrast of the $200-\mathrm{kHz}$ TOF MIEZE signal under configuration (ii). Figure 8(a) shows the contour map of the contrast as a function of the neutron wavelength and $\Omega$. For all wavelengths, the contrast exhibited the maximum value at $\Omega=0$. Figure $8(\mathrm{~b})$ shows the normalized contrast as a function of $\Omega$ for representative neutron wavelengths $\lambda=$ $0.4,0.8,1.2 \mathrm{~nm}$. The open markers indicate the data points detuned via the spatial displacements, and the closed markers indicate the points detuned via the magnetic fields. For longer wavelengths, the contrast decreases more rapidly with an increase in $\Omega$. The difference in the contrast reduction rate can be attributed to the wavelength dependency of the pulse width, which is determined by the moderator characteristics $[49,50]$. Considering the wide margin of the echo condition owing to short pulsed beams, the use of the stacked-type detector consisting of multiple thin conversion layers [51] in the TOF MIEZE technique is of great statistical advantage.

\section{E. Phase correction for scattering experiments}

Even though the apparatus is aligned in the echo condition for the incident beam, the neutrons scattered from the sample may have different trajectories and hence the echo condition can break locally. From Eq. (20), with a path-length difference $\Delta L_{s d}$, the oscillating part of MIEZE signal, $\cos \left[\phi\left(v, t_{d}\right)\right]$, becomes

$$
\begin{aligned}
\cos \left[\omega_{M} t_{d}-\Omega \frac{L_{0 d}}{v}\right] & =\cos \left[\omega_{M} t_{d}-\Delta \phi\right] \\
& =\cos \left[\omega_{M}\left(t_{d}-\Delta t\right)\right]
\end{aligned}
$$

with

$$
\Delta \phi=\frac{\omega_{M}}{v} \Delta L_{s d}=\omega_{M} \Delta t, \quad \text { and } \quad \Delta t=\frac{\Delta L_{s d}}{v} .
$$
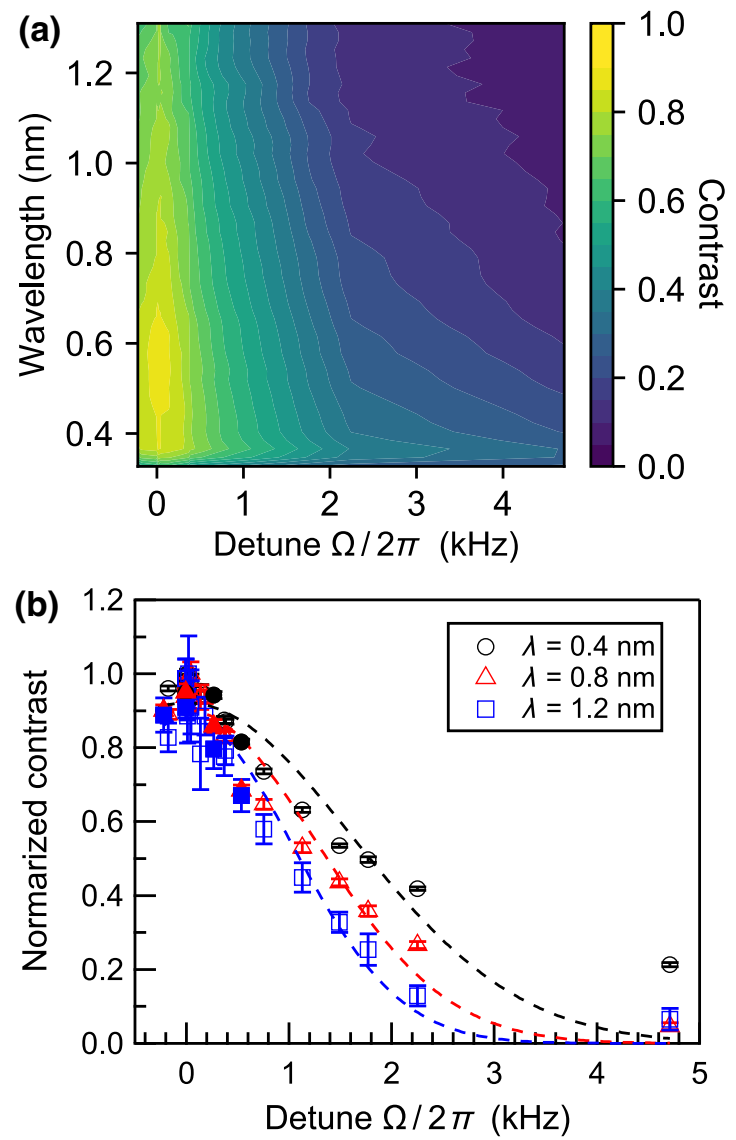

FIG. 8. (a) Contrast of a $200-\mathrm{kHz}$ TOF MIEZE signal as a function of the neutron wavelength and $\Omega$. The data corresponds to configuration (ii). (b) Normalized contrast as a function of $\Omega$ sliced with neutron wavelengths $\lambda=0.4,0.8,1.2 \mathrm{~nm}$. The maximum values are normalized to unity for each wavelength. The open and closed symbols represent the data points detuned via the spatial displacement and magnetic fields, respectively. The dashed lines indicate the best-fit curves by the Gaussian function.

This phase shift is the same as that used in Refs. [35,36]. Because $\Delta L_{s d}$ depends on the position $(x, z)$ of the detector plane, $\Delta \phi$ varies depending on the position and neutron velocity. To draw an $\mathcal{I}(Q, \tau)$ curve for a given $Q$ value, the neutron counts at different positions are collected and filled into a time histogram, which shows the MIEZE signal. In this process, the summation of signals with the different phase shifts $\Delta \phi(v, x, z)$ causes a contrast reduction, which does not reflect the true $\mathcal{I}(Q, \tau)$. Two correction processes for the position-dependent phase have been applied in the MIEZE spectrometer named RESEDA at MLZ [52]. One is the time-shifting method and the other relies on mapping the phase shift in advance for an elastic scatterer. As described below, the correction method by time-shifting is generally straightforward and efficient for the eventbased data-acquisition system, which is widely employed in pulsed neutron-source facilities. Here, we consider the phase-shift problem on a layout of small-angle neutron 

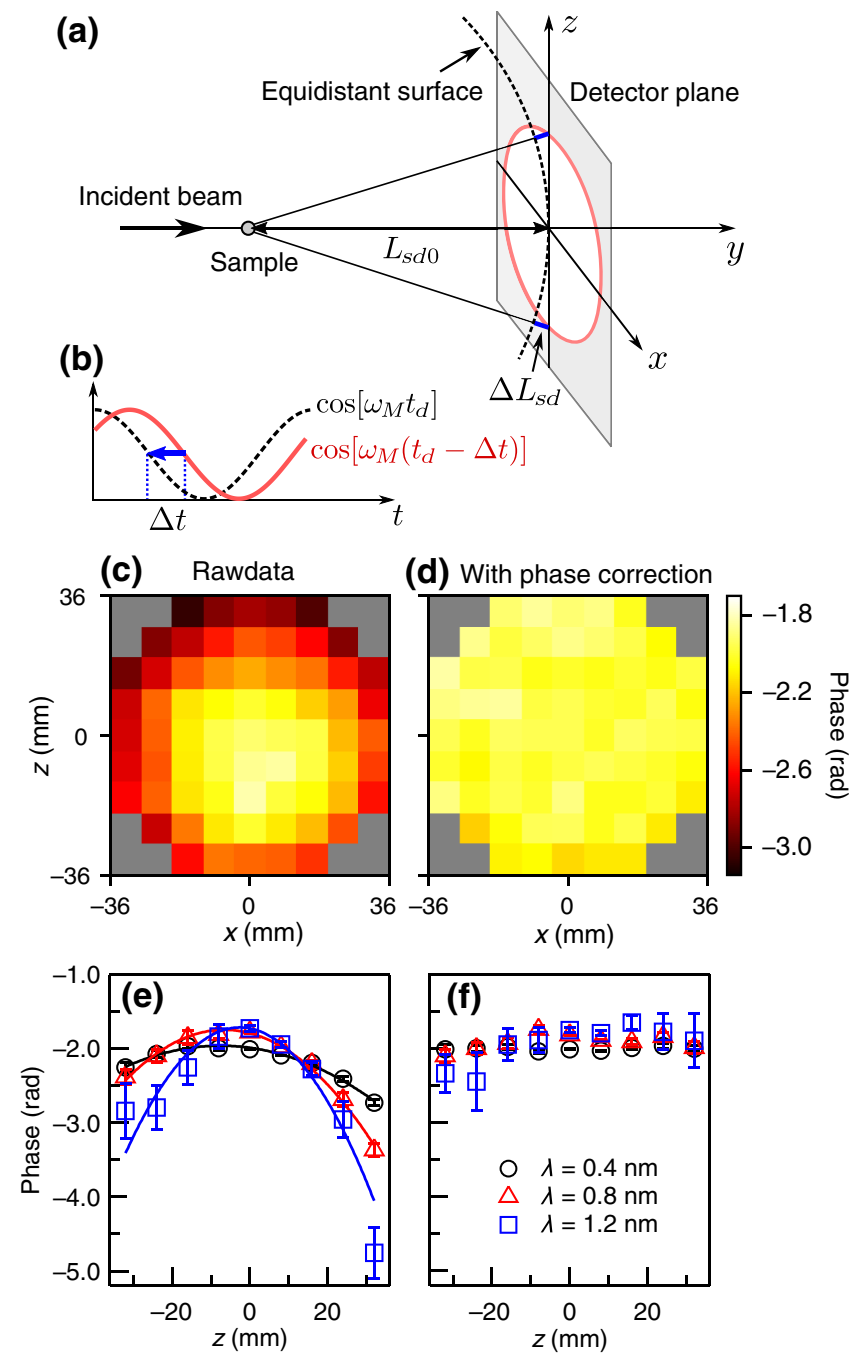

FIG. 9. (a) Schematic layout of a scattering experiment. (b) Phase shift between the actual MIEZE signal (solid line) and that in the case of $\Delta L_{s d}=0$ (dashed line). (c),(d) Phase maps on the detector plane of the $200-\mathrm{kHz}$ MIEZE signal for neutron wavelength $\lambda=0.8 \mathrm{~nm}$ without and with the phase correction, respectively. The two-dimensional histogram consists of spatial bins with an area of $8 \times 8 \mathrm{~mm}^{2}$. The gray hatched bins indicate the region outside of the detector area. (e),(f) $z$ position dependence of phase at the center line $(x=0)$ without and with the phase correction, respectively, for neutron wavelengths $\lambda=0.4 \mathrm{~nm}$ (circles), $0.8 \mathrm{~nm}$ (triangles), and $1.2 \mathrm{~nm}$ (squares). The solid lines in (e) represent the best-fit curves to a quadratic polynomial of the position $z$.

scattering, as shown in Fig. 9(a), which is a likely target of MIEZE spectroscopy. We assume that the detector plane is flat and perpendicular to the incident-beam direction, and the sample is regarded as a point scatterer. In this case, the path-length deviation is given by

$$
\Delta L_{s d}(x, z)=\sqrt{L_{s d 0}^{2}+x^{2}+z^{2}}-L_{s d 0} \simeq \frac{x^{2}+z^{2}}{2 L_{s d 0}},
$$

where $L_{s d 0}$ is the shortest distance between the sample and the detector. The approximation is valid under $\sqrt{x^{2}+z^{2}} \ll L_{s d 0}$. In the perpendicular configuration, $\Delta L_{s d}(x, z) \geq 0, \Delta \phi \geq 0$, and $\Delta t \geq 0$. To reproduce the MIEZE signal on the virtual equidistant surface in the echo condition, the observed $t$ must be shifted to $t-\Delta t$ and filled into a histogram [see Fig. 9(b)]. With the eventdata format, where the detection time $t$ (TOF) and position $(x, z)$ are recorded for each detection event, it is possible to calculate $\Delta t(v, x, z)$ for each neutron before making a time histogram. Therefore, the resolution of $\Delta t$ is free from the constraint by fixed time binning. This is different from the data-acquisition system in which data are recorded in a premade histogram with a fixed binning. Figures 9(c)-9(f) show the experimental data of the observed phase $[(\mathrm{c}, \mathrm{e})]$ and the corrected phase $[(\mathrm{d}, \mathrm{f})]$ of the MIEZE signal in the setup of $L_{s d 0}=1.34 \mathrm{~m}$ and $f_{M}=200 \mathrm{kHz}$. Polycrystalline diamond powder (Microdiamant AG) is used as the elastic sample. The scattered neutrons irradiated the detector with a diameter of $8 \mathrm{~cm}$. The $z$-position dependence at the center line $(x=0)$ shown in Fig. 9(e) can be described by the quadratic functions of $z$ according to Eqs. (33) and (34) for $x=0$. After the phase correction is applied, the phase is much less dependent on position than the raw data for all wavelengths [compare Figs. 9(e) and 9(f)]. The maximum phase shift from the average phase is suppressed at most approximately $0.5 \mathrm{rad}$ in the worst case of $\lambda=1.2 \mathrm{~nm}$. From the identity $[\cos \phi+\cos (\phi+\Delta \phi)] / 2=\cos (\Delta \phi / 2) \cos (\phi+$ $\Delta \phi / 2)$, the contrast reduction factor caused by $\Delta \phi=$ 0.5 is estimated to be $\cos (\Delta \phi / 2) \approx 0.97$, which is not a predominant error in practical measurements. The phase correction method is significant for longer Fourier times and larger scattering angles because the phase shift is more prominent in these cases. The successful phase correction permits the wide coverage of detectors and maximizes the benefit of the broad wavelength band of pulsed beams.

\section{CONCLUSION}

In this work, we propose the introduction of a detuning parameter $\Omega$ as an indicator for the magnitude of deviation from the spin-echo condition, caused by the presence of magnetic fields and spatial displacements. It is demonstrated that the velocity-dependent phase shift emerges as a frequency shift $\Omega$ of the TOF MIEZE signals, and the shift equals the derivative of the phase with respect to the TOF, $\partial \phi / \partial t_{0 d}$. The observed frequency shift and phase derivative exhibited a good agreement with the $\Omega$ values calculated analytically. These findings enable the accurate and precise tuning of a MIEZE system and contribute to the data analysis of the spectroscopy. For example, the linear TOF dependency of the phase can help establish reliable 
constraints in the signal fitting of MIEZE oscillations. Moreover, understanding the phase shift of TOF MIEZE signals is critical to realize the phase correction in a large-area detector.

\section{ACKNOWLEDGMENTS}

We wish to thank H. Ohshita for the information on neutron-detector efficiency. We also thank S. Satoh for the valuable help in the operation of detectors. T.O. acknowledges the fruitful discussions with T. Nakajima. We are grateful to N. Achiwa for the constant encouragement. The neutron-scattering experiment is approved by the Neutron Scattering Program Advisory Committee of IMSS, KEK (Proposals No. 2014S07 and No. 2019S07). Certain device developments are supported by the program of the Development of System and Technology for Advanced Measurement Analysis (SENTAN), JST, the Photon and Quantum Basic Research Coordinated Development Program, MEXT Japan, and the Neutron Scattering Program Advisory Committee of IMSS, KEK (Proposals No. 2009S07 and No. 2014S07). This work is partly supported by JSPS KAKENHI Grants No. JP17K14133, No. JP19K20601, and No. JP19H01856.

[1] F. Mezei, Neutron spin echo: A new concept in polarized thermal neutron techniques, Z. Phys. 255, 146 (1972).

[2] D. Richter, M. Monkenbusch, A. Arbe, and J. Colmenero, Neutron Spin Echo in Polymer Systems (Springer-Verlag, Berlin, Heidelberg, 2005), p. 1.

[3] F. Mezei, Critical dynamics in isotropic ferromagnets, J. Magn. Magn. Mater. 45, 67 (1984).

[4] R. Gähler, R. Golub, and T. Keller, Neutron resonance spin echo-a new tool for high resolution spectroscopy, Phys. B 180, 899 (1992).

[5] R. Gähler and R. Golub, A high resolution neutron spectrometer for quasielastic scattering on the basis of spin-echo and magnetic resonance, Z. Phys. B 65, 269 (1987).

[6] R. Georgii, G. Brandl, N. Arend, W. Häußler, A. Tischendorf, C. Pfleiderer, P. Böni, and J. Lal, Turn-key module for neutron scattering with sub-micro-eV resolution, Appl. Phys. Lett. 98, 073505 (2011).

[7] H. Hayashida, M. Hino, M. Kitaguchi, N. Achiwa, and Y. Kawabata, A new MIEZE technique for investigating relaxation of magnetic nanoparticles, Nucl. Instrum. Methods A 600, 56 (2009).

[8] C. Franz, O. Soltwedel, C. Fuchs, S. Säubert, F. Haslbeck, A. Wendl, J. K. Jochum, P. Böni, and C. Pfleiderer, The longitudinal neutron resonant spin echo spectrometer RESEDA, Nucl. Instrum. Methods A 939, 22 (2019).

[9] C. Franz, S. Säubert, A. Wendl, F. X. Haslbeck, O. Soltwedel, J. K. Jochum, L. Spitz, J. Kindervater, A. Bauer, P. Böni, and C. Pfleiderer, MIEZE neutron spin-echo spectroscopy of strongly correlated electron systems, J. Phys. Soc. Jpn. 88, 081002 (2019).
[10] J. Kindervater, S. Säubert, and P. Böni, Dipolar effects on the critical fluctuations in Fe: Investigation by the neutron spin-echo technique MIEZE, Phys. Rev. B 95, 014429 (2017).

[11] S. Säubert, J. Kindervater, F. Haslbeck, C. Franz, M. Skoulatos, and P. Böni, Dipolar interactions in Fe: A study with the neutron Larmor precession technique MIEZE in a longitudinal field configuration, Phys. Rev. B 99, 184423 (2019).

[12] C. Pappas, L. J. Bannenberg, E. Lelièvre-Berna, F. Qian, C. D. Dewhurst, R. M. Dalgliesh, D. L. Schlagel, T. A. Lograsso, and P. Falus, Magnetic Fluctuations, Precursor Phenomena, and Phase Transition in $\mathrm{MnSi}$ under a Magnetic Field, Phys. Rev. Lett. 119, 047203 (2017).

[13] J. Kindervater, I. Stasinopoulos, A. Bauer, F. X. Haslbeck, F. Rucker, A. Chacon, S. Mühlbauer, C. Franz, M. Garst, D. Grundler, and C. Pfleiderer, Weak Crystallization of Fluctuating Skyrmion Textures in MnSi, Phys. Rev. X 9, 041059 (2019).

[14] T. Nakajima, T. Oda, M. Hino, H. Endo, K. Ohishi, K. Kakurai, A. Kikkawa, Y. Taguchi, Y. Tokura, and T. Arima, Crystallization of magnetic skyrmions in $\mathrm{MnSi}$ investigated by MIEZE-type neutron spin echo spectroscopy (2020), submitted.

[15] B. Farago, Neutron Spin Echo Spectroscopy. Basics, Trends and Applications (Springer-Verlag, Heidelberg, 2003), p. 15.

[16] S. Tasaki, Y. Kawabata, M. Hino, R. Maruyama, and T. Ebisawa, Neutron spin echo spectrometers for J-PARC with resonance spin flippers, J. Neutron Res. 13, 107 (2005).

[17] M. Monkenbusch, M. Ohl, D. Richter, C. Pappas, G. Zsigmond, K. Lieutenant, and F. Mezei, Aspects of neutron spin-echo spectrometer operation on a pulsed source, J. Neutron Res. 13, 63 (2005).

[18] Y. Kawabata, M. Hino, M. Kitaguchi, H. Hayashida, S. Tasaki, T. Ebisawa, D. Yamazaki, R. Maruyama, H. Seto, M. Nagao, and T. Kanaya, Neutron resonance spin echo and MIEZE spectrometer development project in Japan, Phys. B 385-386, Part 2, 1122 (2006).

[19] P. A. Zolnierczuk, O. Holderer, S. Pasini, T. Kozielewski, L. R. Stingaciu, and M. Monkenbusch, Efficient data extraction from neutron time-of-flight spin-echo raw data, J. Appl. Crystallogr. 52, 1022 (2019).

[20] M. Bleuel, M. Broll, E. Lang, K. Littrell, R. Gähler, and J. Lal, First tests of a MIEZE (modulated intensity by zero effort)-type instrument on a pulsed neutron source, Phys. B 371, 297 (2006).

[21] G. Brandl, J. Lal, J. Carpenter, L. Crow, L. Robertson, R. Georgii, P. Böni, and M. Bleuel, Tests of modulated intensity small angle scattering in time of flight mode, Nucl. Instrum. Methods A 667, 1 (2012).

[22] T. Oda, M. Hino, M. Kitaguchi, H. Filter, P. Geltenbort, and Y. Kawabata, Towards a high-resolution TOF-MIEZE spectrometer with very cold neutrons, Nucl. Instrum. Methods A 860, 35 (2017).

[23] N. Geerits, S. R. Parnell, M. A. Thijs, A. A. van Well, C. Franz, A. L. Washington, D. Raspino, R. M. Dalgliesh, and J. Plomp, Time of flight modulation of intensity by zero effort on larmor, Rev. Sci. Instrum. 90, 125101 (2019). 
[24] T. Oda, M. Hino, M. Kitaguchi, P. Geltenbort, and Y. Kawabata, Pulsed neutron time-dependent intensity modulation for quasi-elastic neutron scattering spectroscopy, Rev. Sci. Instrum. 87, 105124 (2016).

[25] G. Badurek, H. Rauch, and J. Summhammer, TimeDependent Superposition of Spinors, Phys. Rev. Lett. 51, 1015 (1983).

[26] R. Golub, R. Gähler, and T. Keller, A plane-wave approach to particle-beam magnetic-resonance, Am. J. Phys. 62, 779 (1994).

[27] M. Köppe, M. Bleuel, R. Gähler, R. Golub, P. Hank, T. Keller, S. Longeville, U. Rauch, and J. Wuttke, Prospects of resonance spin echo, Phys. B 266, 75 (1999).

[28] D. Yamazaki, A neutron spin interferometer using two rf$\pi / 2$ flippers, Nucl. Instrum. Methods A 488, 623 (2002).

[29] S. Sponar, J. Klepp, R. Loidl, S. Filipp, G. Badurek, Y. Hasegawa, and H. Rauch, Coherent energy manipulation in single-neutron interferometry, Phys. Rev. A 78, 061604(R) (2008).

[30] S. V. Grigoriev, W. H. Kraan, F. M. Mulder, and M. T. Rekveldt, Neutron-wave-interference experiments with two resonance coils, Phys. Rev. A 62, 063601 (2000).

[31] J. Zhao, W. A. Hamilton, S.-W. Lee, J. L. Robertson, L. Crow, and Y. W. Kang, Neutron intensity modulation and time-focusing with integrated larmor and resonant frequency techniques, Appl. Phys. Lett. 107, 113508 (2015).

[32] G. L. Squires, Introduction to the Theory of Thermal Neutron Scattering (Cambridge University Press, Cambridge, 2012), 3rd ed.

[33] W. Besenböck, R. Gähler, P. Hank, R. Kahn, M. Köppe, C. H. de Novion, W. Petry, and J. Wuttke, First scattering experiment on MIEZE: A fourier transform time-of-flight spectrometer using resonance coils, J. Neutron Res. 7, 65 (1998).

[34] F. Mezei, Neutron Spin Echo (Springer-Verlag, Heidelberg, 1980), Chap. 1.

[35] H. Hayashida, M. Hino, M. Kitaguchi, Y. Kawabata, and N. Achiwa, A study of resolution function on a MIEZE spectrometer, Meas. Sci. Technol. 19, 034006 (2008).

[36] G. Brandl, R. Georgii, W. Häeußler, S. Mühlbauer, and P. Böni, Large scales-long times: Adding high energy resolution to sans, Nucl. Instrum. Methods A 654, 394 (2011).

[37] T. Weber, G. Brand, R. Georgii, W. Häußler, S. Weichselbaumer, and P. Böni, Monte-Carlo simulations for the optimisation of a TOF-MIEZE instrument, Nucl. Instrum. Methods A 713, 71 (2013).

[38] N. Martin, On the resolution of a MIEZE spectrometer, Nucl. Instrum. Methods A 882, 11 (2018).

[39] K. Nakajima et al., Materials and life science experimental facility (MLF) at the Japan proton accelerator research complex II: Neutron scattering instruments, Quantum Beam Sci. 1, 9 (2017).

[40] R. Kajimoto, T. Yokoo, M. Nakamura, Y. Kawakita, M. Matsuura, H. Endo, H. Seto, S. Itoh, K. Nakajima, and S. Ohira-Kawamura, Status of neutron spectrometers at J-PARC, Phys. B 562, 148 (2019).

[41] H. Seto, S. Itoh, T. Yokoo, H. Endo, K. Nakajima, K. Shibata, R. Kajimoto, S. Ohira-Kawamura, M. Nakamura, Y. Kawakita, H. Nakagawa, and T. Yamada, Inelastic and quasi-elastic neutron scattering spectrometers in J-PARC, Biochim. Biophys. Acta (BBA) 1861, 3651 (2017).

[42] H. Takada, K. Haga, M. Teshigawara, T. Aso, S.-I. Meigo, H. Kogawa, T. Naoe, T. Wakui, M. Ooi, M. Harada, and M. Futakawa, Materials and life science experimental facility at the Japan proton accelerator research complex I: Pulsed spallation neutron source, Quantum Beam Sci. 1, 8 (2017).

[43] M. Hino, T. Oda, N. L. Yamada, H. Endo, H. Seto, M. Kitaguchi, M. Harada, and Y. Kawabata, Supermirror neutron guide system for neutron resonance spin echo spectrometers at a pulsed neutron source, J. Nucl. Sci. Technol. 54, 1223 (2017).

[44] K. Hirota, T. Shinohara, K. Ikeda, K. Mishima, T. Adachi, T. Morishima, S. Satoh, T. Oku, S. Yamada, H. Sasao, J. Suzuki, and H. M. Shimizu, Development of a neutron detector based on a position-sensitive photomultiplier, Phys. Chem. Chem. Phys. 7, 1836 (2005).

[45] S. Satoh, S. Muto, N. Kaneko, T. Uchida, M. Tanaka, Y. Yasu, K. Nakayoshi, E. Inoue, H. Sendai, T. Nakatani, and T. Otomo, Development of a readout system employing high-speed network for J-PARC, Nucl. Instrum. Methods A 600, 103 (2009).

[46] R. Maruyama, T. Ebisawa, S. Tasaki, M. Hino, M. Takeda, T. Kawai, Y. Kawabata, and K. Sakai, A resonance neutronspin flipper for neutron spin echo at pulsed sources, Phys. B 335, 238 (2003).

[47] W. Häußler and U. Schmidt, Effective field integral subtraction by the combination of spin echo and resonance spin echo, Phys. Chem. Chem. Phys. 7, 1245 (2005).

[48] M. Krautloher, J. Kindervater, T. Keller, and W. Häußler, Neutron resonance spin echo with longitudinal DC fields, Rev. Sci. Instrum. 87, 125110 (2016).

[49] J-PARC.jp, Technical details of materials and life science experimental facility, http://j-parc.jp/researcher/MatLife/en/ instrumentation/ns3.html (2004).

[50] M. Harada, N. Watanabe, M. Teshigawara, T. Kai, F. Maekawa, T. Kato, and Y. Ikeda, in Proceedings of the 17th Meeting of the International Collaboration on Advanced Neutron Sources (ICANS-XVII), 700 (2006), LA-UR-063904.

[51] M. Köhli, F. Allmendinger, W. Häußler, T. Schröder, M. Klein, M. Meven, and U. Schmidt, Efficiency and spatial resolution of the CASCADE thermal neutron detector, Nucl. Instrum. Methods A 828, 242 (2016).

[52] A. Schober, A. Wendl, F. X. Haslbeck, J. K. Jochum, L. Spitz, and C. Franz, The software package MIEZEPY for the reduction of MIEZE data, J. Phys. Commun. 3, 103001 (2019). 